\title{
Trainer-exerciser relationship: The congruency effect on exerciser psychological needs using response surface analysis
}

\author{
Filipe Rodrigues $^{1,2,3,4}$ (D) | Luc G. Pelletier ${ }^{5} \mid$ Meredith Rocchi $^{6} \quad$ Henrique P. Neiva ${ }^{1,3}$

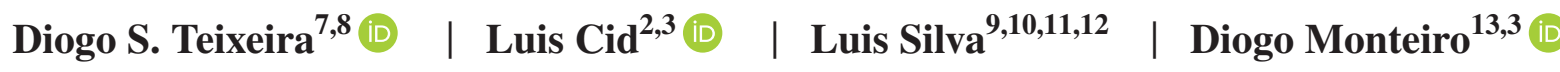 \\ ${ }^{1}$ Department of Sports Science, University of Beira Interior (UBI), Covilhã, Portugal \\ ${ }^{2}$ Sport Science School of Rio Maior (ESDRM-IPSantarém), Rio Maior, Portugal \\ ${ }^{3}$ Research Center in Sport, Health and Human Development (CIDESD), Vila Real, Portugal \\ ${ }^{4}$ Life Quality Research Center (CIEQV), Rio Maior, Portugal \\ ${ }^{5}$ School of Psychology, University of Ottawa, Ottawa, ON, Canada \\ ${ }^{6}$ Department of Communication, University of Ottawa, Ottawa, ON, Canada \\ ${ }^{7}$ Lusófona University of Humanities and Technology (ULHT), Lisbon, Portugal \\ ${ }^{8}$ Center for the Study of Human Performance (CIPER), Lisbon, Portugal \\ ${ }^{9}$ Kinesiolab, Instituto Piaget, Lisbon, Portugal \\ ${ }^{10}$ Department of Biomechanics, University of Nebraska Omaha, Omaha, NE, USA \\ ${ }^{11}$ Neuromuscular Research Lab, University of Lisbon Faculty of Human Kinetics, Lisbon, Portugal \\ ${ }^{12}$ Centro Lusíada de Investigação em Serviço Social e Intervenção Social, Lusíada University of Lisbon, Lisbon, Portugal \\ ${ }^{13}$ Department of Human Kinetics, ESECS, Polytechnique of Leiria, Leiria, Portugal
}

\section{Correspondence}

Diogo Monteiro, Department of Human

Kinetics, ESECS, Polytechnique of Leiria,

Leiria, Portugal, Campus 1 Rua Dr. João

Soares Apt 4045, 2411-901

Email: diogo.monteiro@ipleiria.pt

\section{Funding information}

LC, HPN, and DM were supported by national funds through the Portuguese

Foundation for Science and Technology, IP, under the project UID04045/2020.
Perceptions of fitness trainers' need-supportive and need-thwarting behaviors have been shown to impact exercisers' psychological need satisfaction and frustration. Currently, it is unknown whether an agreement or disagreement between exercisers' and fitness trainers' reported perceptions of these behaviors leads to the satisfaction and/or frustration of psychological needs. Based on self-determination theory, the present study examined the effect of congruency between fitness trainers' and exercisers' perceptions of need-supportive and need-thwarting interpersonal behaviors on basic psychological need satisfaction and frustration. A sample of 130 fitness trainers (43 females; $M_{\text {age }}=31.58 \pm 7.65$ ) and a total of 640 gym exercisers (350 females; $\left.\mathrm{M}_{\text {age }}=34.23 \pm 11.59\right)$ participated in this study. Findings suggested that the majority of fitness trainers tended to over-report their use of need-supportive behavior and under-report their need-thwarting behaviors. Results showed that when there was congruency between fitness trainers' reported use and exercisers' perception of interpersonal behaviors, basic need satisfaction tended to increase. This effect was greater for exercisers that rated their respective fitness trainer high on relatedness support. Fitness trainers should be self-aware of their interpersonal behaviors when engaging with exercisers and interventions based on self-determination theory could serve as a promising avenue to improve the quality of exercisers' experience. 


\section{K E Y W O R D S}

basic needs, exercisers, fitness professionals, interpersonal behaviors, response surface analysis

\section{$1 \mid$ INTRODUCTION}

Motivation has been identified as a key driver of human engagement in physical exercise. Individuals who engage in exercise due to their own volition and intrinsic motivation persist more in the long term. ${ }^{1}$ Contrarily, those who rely on external or self-imposed pressures are less likely to participate in continuous or long-term physical exercise engagement. ${ }^{2}$ In this regard, exercisers often rely on fitness trainers (eg, group fitness leaders, fitness professionals) to guide and motivate them in their exercise participation. Through their interactions with exercisers, fitness trainers create contexts that lead to increased satisfaction, better performances, and more persistence over time. ${ }^{3}$ Alternatively, they can also create contexts that may have negative effects and undermine the individual's exercise experience which can lead to decreased satisfaction and dropout. The quality of the exerciser experience is therefore highly contingent on how they perceive their fitness trainer as either a supportive or controlling figure.

Most empirical studies to date have only examined interpersonal behaviors from the perspective of exercisers. ${ }^{2}$ The fitness trainers' perception of their own behaviors, however, should arguably also be assessed to determine how it relates to exercisers' motivation. A question in the current study is the extent to which the congruency effect between one's own behaviors (ie, fitness trainers' self-reported evaluation of their behaviors) and perception from others (ie, exercisers' perceptions of fitness trainers' behaviors) are associated with optimal or non-optimal motivation for exercise. The current study uses a response surface analysis (RSA) approach to expand existing literature assessing the congruency hypothesis that the agreement between perceived behaviors should have a positive (or negative) effect on an outcome variable. $^{4}$

\section{1 | Motivational framework}

Self-determination (SDT) is a broad theory of human motivation that has both basic and applied research applications. ${ }^{5}$ SDT defines motivation in terms of autonomous and controlled orientations. If an individual has autonomous motivation for an activity, where they engage in the activity because they want to, this is associated with positive and adaptive outcomes such as persistence or commitment. If an individual has controlled motivation, where they engage in an activity because they have to, this is associated with negative outcomes such as burnout or dropout. ${ }^{6}$ Extensive research under the SDT framework has aimed to determine what promotes and undermines autonomous motivation. This large body of research has shown that interpersonal behaviors can have positive or negative effects on motivation and the subsequent quality of behavior engagement. ${ }^{2}$

SDT argues that all humans possess three basic psychological needs that are essential to an individual's growth, integrity, and optimal functioning. ${ }^{5}$ The need for autonomy refers to the need for volition or to do what one wants. In the context of exercise, if the need for autonomy is fulfilled, the exerciser endorses their own actions and chooses to engage in exercises they enjoy. The need for competence is the need to feel effective and capable in the activities the individual chooses to engage in. In the exercise context, individuals who feel they are developing their skills at a specific exercise, increasing their working load, and/or enduring higher training intensities will feel their need for competence is satisfied. Finally, the need for relatedness touches upon a sense of connectedness with others. In the exercise context, individuals need to feel that they matter in some way, experience care from other exercisers and fitness trainers, or that they contribute to the group. When these three basic psychological needs are satisfied, an individual will thrive; whereas when the needs are frustrated, an individual experiences a degradation in functioning. ${ }^{7}$

It should be noted that need satisfaction and frustration are distinct constructs since low levels of need satisfaction are not necessarily related to high levels of need frustration. Specifically, Vansteenkiste and colleagues ${ }^{8}$ consider satisfaction and frustration as distinct predictors, with the presence of frustration having a stronger and more menacing influence on non-optimal consequences than the mere absence of need satisfaction. As such, autonomy frustration defines the feeling of external or internal controlled forces, with no willingness to carry out the behavior. Competence frustration involves having doubts or feeling ineffective at achieving desired outcomes. Finally, relatedness frustration involves the feeling of social exclusion and loneliness. The distinction between need satisfaction and frustration has provided a more advanced understanding of both optimal functioning and non-optimal functioning of human behavior. ${ }^{9}$ Thus, basic psychological need satisfaction has been associated with adaptive outcomes such as autonomous motivation and exercise persistence, whereas frustration has been associated with maladaptive consequences such as controlled motivation and lower intentions to continue exercising. ${ }^{2}$ 


\section{2 | Need-supportive and need- thwarting behaviors}

The satisfaction or frustration of psychological needs is dependent upon how individuals perceive the interpersonal behaviors of others within their social environment. ${ }^{7}$ That is, exercisers' satisfaction or frustration of their basic psychological needs is dependent on how they perceive need-supportive and need-thwarting behaviors from others, including fitness trainers, when they exercise. According to SDT, there are specific types of interpersonal behaviors that are directly associated with each of the three basic psychological needs. Needsupportive behaviors promote need satisfaction, whereas need-thwarting behaviors lead to need frustration. ${ }^{5}$

Autonomy-supportive behaviors begin with understanding an exercisers' internal frame of reference in which they perceive a sense of volitional choice. In this sense, fitness trainers can support autonomy by providing a rationale for requested exercises, encouraging initiation and expressing exerciser ownership, or providing task involvement. ${ }^{10}$ Competence supportive behaviors involve giving positive feedback aimed at mastery or increased skills, where they can encourage learning and show they believe individuals can meet their goals. Thus, fitness trainers can design training programs so that mastery is the dominant experience, providing structure and optimal challenges in which exercisers can regularly succeed. ${ }^{11}$ Relatedness supportive behaviors require providing emotional support and showing connection and care. In this regard, fitness trainers convey respect and support for exercisers so that they feel valued and significant in their training program, as well as provide warmth and inclusion so that the exerciser feels connected. ${ }^{11}$

Autonomy thwarting behaviors are coercive in nature and are defined by the use of controlling language in which individuals feel pressured to behave in a way that is not their volition. ${ }^{12}$ Fitness trainers can engage in controlling behaviors or use rewards contingent on exercisers' results by means of pressured feedback. Competence thwarting behaviors are defined as expressions of behaviors that emphasize doubt or in which feedback is provided in an evaluative, rather than informational, manner. Here, fitness trainers could thwart competence by highlighting when exercisers make a mistake or focusing praise on comparisons with others rather than effort and accomplishments. Last, relatedness thwarting encompasses showing feelings of rejection and discredit, being cold or distant. These behaviors are often perceived as lacking connection, or viewed as manipulative or without concern for well-being and self-worth. ${ }^{2}$

\section{3 | Self-other perception of interpersonal behaviors}

When exercisers perceive need-supportive behaviors from fitness trainers, their basic psychological needs are satisfied and they experience autonomous motivation where they enjoy exercising, they want to improve their skills and fitness, and they choose to train continuously without external contingencies. ${ }^{2}$ On the other hand, when exercisers perceive need-thwarting behaviors, their basic psychological needs are more likely to be frustrated and they experience controlled motivation where they feel coerced or pressured to exercise, they train to obtain external rewards or to avoid disappointing others, and are more susceptible to dropping out. ${ }^{13}$

The research on interpersonal behaviors has traditionally relied on a one-way perspective (either self-reported or other-reported) to determine how the interpersonal behavior of a person in a position of leadership (ie, fitness trainer, coach) is linked to a given outcome. ${ }^{2}$ For example, numerous studies in the sport and exercise domains have examined the outcome of interpersonal behavior by measuring athletes' or exercise participants' perceptions of their coach's or fitness trainer's behavior and its relationship to psychological needs, motivation, and outcomes (other-reported studies). ${ }^{10,14}$ More recently, researchers have aimed to understand the antecedents of interpersonal behavior by asking coaches or fitness trainers to report on their own experiences, as well as their interpersonal behavior styles (self-report studies). ${ }^{15,16}$ This research has generally found that coaches and trainers who are autonomous in their own contexts are more likely to report engaging in supportive interpersonal behavior, whereas those who feel controlled are more likely to report thwarting behavior. A recent study conducted by Rocchi and Pelletier ${ }^{17}$ has raised some limitations with the use of these traditional linear approaches.

The first limitation is that the assumption underlying this research is that when a person in a leadership position self-reports their use of interpersonal behaviors, these self-reports align with others' perceptions of these same behaviors. For example, if a fitness trainer reported that they engage in need-supportive behavior, their exerciser would agree. Recent research, however, suggests that it may not always be the case. In the context of sport, for example, there can be a discrepancy where some coaches have a tendency to over-report their own behavior by evaluating their need-supportive behavior more positively than what is perceived by their athletes. ${ }^{17}$ Coaches may also under-report where they consider themselves to be less need-supportive compared with their athletes' perceptions. In agreement with the SDT framework, ${ }^{5}$ when coaches and athletes' perceptions of need-supportive behaviors were high, athletes experienced higher levels of psychological need satisfaction. As long as the exerciser's perception of their trainer's interpersonal behaviors is favorable, the exerciser will still experience positive outcomes. ${ }^{18}$ However, when Rocchi and Pelletier ${ }^{17}$ examined the consequences of the levels of agreement and discrepant behaviors, athletes with coaches who under-reported their need-supportive behaviors (and over-reported their need-thwarting behaviors) experienced additional need satisfaction and less need 
frustration. In contrast, athletes with coaches who over-reported their need-supportive behaviors (and under-reported their need-thwarting behaviors) perceived less need satisfaction and more need frustration. As such, both the self-reported and other-reported behaviors made a unique contribution to athletes' psychological needs, supporting that both should be assessed.

A second limitation is that when people in leadership positions report their interpersonal behaviors, they typically report how they behave in general, not in regard to how they behave with each of their trainees individually. ${ }^{10}$ This may be problematic because their perceptions of their behavior in general may not be representative of how they interact with each unique individual. For instance, previous research suggests that fitness trainers may be more autonomy-supportive when dealing with exercisers that are autonomously motivated. ${ }^{19}$ Alternatively, fitness trainers may become more need-thwarting when dealing with exercisers that have controlled motivation orientations. ${ }^{17}$ As such, when examining self-reported interpersonal behaviors, it is essential to consider behavior in general, as well as specific interactions with each individual.

These limitations highlight the need to move beyond one-way perspectives of interpersonal behavior and incorporate both self-reported and other-reported indicators in order to better understand the role of interpersonal behavior and its relationship to psychological needs and outcomes. Selfdetermination research has demonstrated that the perception of interpersonal behaviors as measured from the exerciser perspective has consequences for athletes' or exercisers' need satisfaction and frustration. ${ }^{2}$ Yet, we argue that it is also paramount to consider fitness trainers' perception of the same interpersonal behaviors, particularly due to recent research in similar context that has found that coaches and their athletes may differ in their perceptions of the same behaviors. ${ }^{17}$ This is referred to as the congruency hypothesis, that is, examining the agreement/disagreement of ratings between people in authoritarian positions (eg, trainers, fitness professionals, coaches) and their followers (eg, athletes, exercisers). The congruency hypothesis states that an individual's values in an outcome variable are higher when their values in two predictor variables are closer to one another. ${ }^{20}$ The congruency (or incongruency) can vary depending on how leaders and followers rate their perceptions on the same independent variables. It is hypothesized that a strong congruency effect, where leaders and followers report similar ratings, would lead to higher levels on the outcome. ${ }^{4}$ On the other hand, higher incongruency, were leaders report higher or lower ratings compared with followers, would be suggestive of lower ratings on the outcome variable. This type of analytical approach plays a crucial role in person-environment research where a person-centered outcome can be affected not only by the individual's perception of their environment, but the environment also impacts the individual's outcome. This approach has been recently incorporated into research within the sport context. ${ }^{17,21,22}$ Given that coaches typically engage with one specific homogenous group of athletes compared with fitness trainers who interact with consistently different groups of people or individuals, it is not clear if the conclusions drawn from the previous literature apply to the exercise context. From a conceptual perspective, previous studies suggest that trainers may be overly positive when rating their own behaviors, ${ }^{23}$ which could be related to higher ratings of need frustration for their exercisers. ${ }^{17}$ In this case, when fitness trainers report high levels of need-thwarting behaviors compared with the perceptions of their exercisers, the exerciser tends to experiences higher levels of need frustration. Contrarily, when exercisers perceive that their trainer is engaging in need-supportive behaviors, even though the fitness trainer does not rate themselves similarly, exercisers experience increased levels of need satisfaction.

\section{4 | Present research}

This study aimed to address existing limitations and provide an important incremental step forward regarding how exercisers' perceptions of interpersonal behaviors and fitness trainers' self-reported use of the same behaviors related to exercisers' psychological need satisfaction and frustration. Through this, this study extended the findings from existing research on self and other-ratings in the sport context ${ }^{17,21}$ to the exercise context. This study had two objectives. The first was to explore the relationship between fitness trainers' reported use of their interpersonal behaviors and exercisers' perceptions of their fitness trainers' use of the same behaviors. Then, analyze the frequency of discrepancies and agreement of these variables. The second objective was to examine the congruency effect between fitness trainers reported and exercisers' perceived need-supportive and need-thwarting behaviors on exercisers' psychological need satisfaction and frustration using an RSA approach. This study will advance previous research in sport and exercise psychology, as it considers the possible multilevel effect ${ }^{24}$ of trainers and exercisers from different gym facilities. That is, previous studies that have collected data in trainers and athletes from different teams have assumed that the "team" variable would not have implications on the assessment of interpersonal behaviors ${ }^{17}$ or motivational climates, ${ }^{21}$ when in fact there could be some shared variance between teams or structures.

For the first objective, we hypothesized that fitness trainers' use and exercisers' perceptions of interpersonal behaviors would be similarly distributed across three different behavior patterns (ie, over-report, under-report, and agreement behaviors). Although this has not been hypothesized in existing trainer-exerciser research, research in other physical activity-related fields such as sports has found that coaches and athletes were only in agreement in about one third of the 
relationships, where the remaining associations tended to be evenly distributed. ${ }^{17}$ These results are based on the work of Ntoumanis, ${ }^{23}$ where trainers may have an inclination to be overly positive about their behaviors relative to the perceptions of the athletes. For the second objective, in agreement with previous studies, ${ }^{17}$ the congruency effect between fitness trainers' and exercisers' need-supportive and need-thwarting behaviors would lead to increased exerciser psychological need satisfaction and frustration, respectively. This is based on the proposition that when exercisers and trainers agree about their perception of the same interpersonal behaviors, and then, the expected outcomes should be better. ${ }^{17}$ One potential reason for this effect is that exercisers are benefiting not only directly from their perceptions of the trainers' behaviors, but also to some degree indirectly from the supportive engagement endorsed by the trainer. This is based on the assumption that engaging in need-supportive interpersonal behaviors benefits not only the beneficiary, but also the donor. ${ }^{5}$ As such, exercisers experiencing higher levels of supportive interpersonal behaviors may also impact how trainers perceive their own behaviors, creating a loop of engagement in need-supportive and a decline in need-thwarting behaviors.

Over-reported need-supportive and under-reported need-thwarting behaviors by trainers, compared with exercise, would lead to less need satisfaction and more need frustration in exercisers, respectively. ${ }^{17}$ This may be true since some trainers may have an inclination to overestimate how their supportive behaviors relate to what their athletes/ exercisers perceive, and downplay how their need-thwarting behaviors affect the athletes/exercisers. ${ }^{17,23}$ Given that self-absorbed (ie, narcissistic) tendencies may lead to controlling behaviors, ${ }^{23}$ it is expected that this type of disagreement between how the trainers and the exercisers perceived the same interpersonal behaviors should lead to more negative consequences for the exercisers.

Under-reported need-supportive and need-thwarting behaviors by trainers compared with exercisers should lead to increased need satisfaction and need frustration, respectively. This may be true since previous studies found that the athletes' perceptions play a more important role in predicting their psychological needs then coaches' actual behavior. ${ }^{25,26}$ In the situation where the trainers are seemingly under-reporting the levels of their interpersonal behaviors, the exercisers' perceptions should prevail over the ones reported by the trainers.

\section{2 | METHODS}

\subsection{Participants and procedures}

A total of 130 Portuguese fitness trainers (female $=43$; male $=87)$ aged 19 to 45 years $(\mathrm{M}=31.58 ; \mathrm{SD}=7.65)$ participated in this study. According to their academic background, $14.6 \%$ had a trainer certification for exercise prescription; $51.5 \%$ had a bachelor's degree, $30.8 \%$ had a master's degree, and $3.1 \%$ had a doctoral degree. Exercise prescription experience ranged from 6 months to 480 months $(\mathrm{M}=88.54 ; \mathrm{SD}=77.89)$. Fitness trainers were all full-time employees and worked in several different gym roles such as leading group fitness classes and providing exercise prescriptions in the cardio-weightlifting room.

Data from 640 Portuguese exercisers (female $=350$; male $=290)$ aged 18 to 65 years $(M=34.23 ; \mathrm{SD}=11.59)$ were collected for the present study. Exercise experience ranged from 6 to 120 months $(\mathrm{M}=56.34$; $\mathrm{SD}=10.81)$. The number of training sessions per week ranged from 2 to $6(\mathrm{M}=2.67$; $\mathrm{SD}=.76)$ and the training periods lasted between 30 and 90 minutes $(M=60.17$; $\mathrm{SD}=14.98)$. Exercisers were enrolled in fitness group classes such as synchronized activities to music tempo (eg, Pump) and non-synchronized activities (eg, Pilates, Cross-training), and/or in cardio-resistance training which involved lifting weights or treadmill running. All participants had access to a variety of different types of activities and could engage in their preferred activities on any given day.

In order to be eligible for this study, exercisers needed to be at least 18 years of age, have at least six months of exercise experience, train at least 2 times per week, and be part of a fitness trainers' client portfolio over the previous four months. Fitness trainers needed to be full-time workers at a gym during the study period and have been in contact with the exercisers at least 2 times per week over the previous four months.

Before beginning data collection, approval for this study was obtained from the Ethical Committee (registration \# CEUBI-pJ-2018-044:ID683). Data collection procedures were conducted in accordance with the Helsinki Declaration and its later amendments. Researchers contacted several gym managers $(n=10)$ responsible for small fitness centers to grant permission to conduct the present research. At this stage, the objectives of the study were explained and endorsement from each participant was obtained. Next, exercisers were contacted through the reception desk at each gym and asked to participate voluntarily in this study. They were informed about the study objectives and gave their informed consent before participating. Confidentiality was reinforced since we asked the participants to write down the name of the fitness professional acting as the responsible person for their exercise prescription. The exercisers completed the study before their fitness trainers in order to ensure that all exercisers were taken into consideration when the fitness trainers participated in the study. Then, the fitness trainers completed measures of their professional background and their overall perception of interpersonal behaviors. It is worth noting that it is difficult for trainers to isolate their behaviors toward specific members since they regularly interact with the exercisers they train, other gym members, and staff members. Nevertheless, researchers made it clear that the purpose of 
the study was to understand how the fitness trainers engage in interpersonal behaviors with the exercisers that they are responsible for their exercise prescription. There were on average 2.41 ( $\mathrm{SD}=.24)$ exercisers per-instructor. The exercisers-per-instructor range was from three to six exercisers, with four instructors having six exercisers linked to them.

Exercisers and fitness trainers were reminded that the study was voluntary in nature and assured that the IBQ and IBQSelf scores would remain confidential and only used for this study. Collected data were not provided to the gym and health club managers. Similar procedures were used in previous research. ${ }^{17}$ Time taken to complete the survey was approximately 12 minutes for exercisers and 17 minutes for fitness trainers.

\section{2 | Measures}

Exercisers completed the Interpersonal Behavior Questionnaire (IBQ) and fitness trainers completed the IBQSelf in the context of exercise. ${ }^{11}$ The IBQ measures how exercisers perceive need-supportive and need-thwarting behaviors engaged in by their fitness trainer. The IBQ-Self measures how fitness trainers perceive their own behaviors when they interact with an exerciser on the behaviors in general. Both 24-item scales measure all six types of interpersonal behaviors outlined by SDT. That is, 12 items (ie, four items per need for autonomy, competence, and relatedness) representing need-supportive behaviors and 12 items (ie, four items per needs for autonomy, competence, and relatedness) representing need-thwarting behaviors. The participants rated the items on a 7-point scale anchored from "Do not agree at all" to "Totally agree." The IBQ and IBQ-Self have demonstrated acceptable levels of reliability and validity in previous research both in the sport ${ }^{12,15,27}$ and in the exercise contexts. ${ }^{2}$

The Basic Psychological Need Satisfaction and Frustration Scale adapted to the context of exercise ${ }^{28}$ was used to measure exerciser psychological need satisfaction and frustration. This 24-item scale includes 12 items (ie, four items per need for autonomy, competence, and relatedness) representing the satisfaction of needs and 12 items (ie, four items per need for autonomy, competence, and relatedness) representing the frustration of needs. Exercisers responded on a 5-point scale ranging from "totally disagree" to "totally agree" according to their perceived feelings during their training sessions. This measure has provided adequate levels of reliability and validity in previous research in several domains and cultures ${ }^{8,29}$ supporting its use as a measure of need satisfaction and need frustration.

\section{3 | Statistical analysis}

Descriptive statistics for all study variables and bivariate correlations among variables were calculated. Normal distributions were assessed by examining skewness and kurtosis estimates, and acceptable normality was considered using $-2 /+2$ and $-7 /+7$ cutoffs. ${ }^{30}$ Reliability coefficients were observed for all study variables suggesting scores $\geq .70$ as acceptable. ${ }^{31}$ Missing values were treated with full information likelihood estimation. ${ }^{32}$ All descriptive statistics analyses were conducted in Mplus 7.4. ${ }^{33}$

\subsection{1 | Frequencies of predictor discrepancies and agreement}

For objective one, the level of agreement, under-reporting, and over-reporting between fitness trainers' and exercisers' ratings of the six types of interpersonal behaviors were calculated. This was done in order to identify which behaviors between fitness trainers and exercisers were in agreement and which were not (ie, under-report and over-report), as recommended when studying leader-follower perceptual distance, ${ }^{34,35}$ as well as to ensure that the level of discrepancy was sufficient for proceeding with further analysis. The minimum level was set to $10 \%$ discrepancy as suggested in previous studies. ${ }^{35}$

All factors related to interpersonal behaviors were standardized to z-scores $(M=0 ; S D=1)$. Then, exercisers' scores were subtracted from their fitness trainers' scores, since follower perceptions may be the most meaningful measure of leader behavior. ${ }^{36}$ The following calculations were then conducted for each of the six SDT behaviors (eg, exerciser perception of autonomy support and fitness trainer reported use of autonomy support): i) the percentage of behaviors in agreement, defined as less than onehalf standard deviation $(-.5 /+.5)$ between both scores; ii) the percentage of over-reported behaviors, explained by the average scores of fitness trainers' behaviors that were .5 standard deviation above exercisers' perception; and iii) the percentage of under-reported behaviors, defined as the average scores are below on half standard deviation. The one-half deviation criterion was based on previous assumptions, ${ }^{37}$ and the cutoff is considered as a reliable source of group characterization. In the final step, average interpersonal behavior ratings were compared with categorize the behaviors into three groups.

\subsection{2 | Response surface analysis}

For objective two, we followed the analytical approach proposed by several authors ${ }^{4,24}$ and adapted it to our study design. After assessing the level of discrepant and agreement behaviors, several criteria were considered for data preparation. Possible multicollinearity problems were analyzed by using the variance inflation factor (VIF), observing scores $<5$ 
as acceptable. ${ }^{38}$ Additionally, the study needed to be sufficiently powered by having at least 2 to 3 times as many participants as would be needed to detect linear main effects. ${ }^{4}$ In this study, VIF scores varied between 2.89 and 3.45 and the sample was larger than the required minimum, and we moved on to the next stage of RSA.

To account for inter-dependency between exercisers-per-trainers associations and different gym facilities, we employed a multilevel model (exercisers nested within trainers nested within gym facilities) RSA approach. Analyses were performed using the lme 4 package in $\mathrm{R}$ (for more details, see Nestler et $\mathrm{al}^{24}$ ). Multilevel RSA was conducted to examine the influence of each need-supportive and need-thwarting behavior on their respective need considering the multilevel structure inherent in the data set (ie, variance in exercisers nested in trainers, and the nested structure within several gym facilities). This analysis enables researchers to examine the effect of two independent variables on a third dependent variable, retaining information about the differences between variables. Before model estimation, the predictor variables, namely fitness trainers' behaviors $\left(b_{1}\right)$ and exercisers' perceptions of those behaviors $\left(b_{2}\right)$, were centered by the grand mean as recommended by Humberg and colleagues. The centering of predictors ensures that the zero point is common to both predictor variables, a prerequisite for examining congruency hypotheses and it reduces possible multicollinearity issues. ${ }^{39}$ Thereafter, the squared terms of the centered variables $\left(b_{3}\right.$ and $\left.b_{4}\right)$ and the interaction term $\left(b_{5}\right)$ were added to the data set. If the predictors explained significant variance in the outcome variable, then further analyses were justified.

Since, in applied sciences such as exercise psychology, estimating the full variance-covariance may be problematic, random slopes were removed as proposed in the literature $^{4}$ and we moved forward with examining the fixed effects of the multilevel RSA parameters. The reason for this is that this study was interested in the within-group estimates and their interactions controlling for any dependencies of the gym facilities variable that remained from using this group-mean-centering strategy as proposed by Preacher, Zhang, and Zyphur. ${ }^{40}$ Estimated regression coefficients from the multilevel RSA were used to calculate four surface test values for the average response surface across higher level units $\left(a_{1}-a_{4}\right)$ that are plotted in graphs and interpreted according to the slopes and curvature of the lines. The first line runs diagonally from the nearest to the farthest corners of the graph. This is called the line of perfect agreement. The response surface $a_{1}\left(b_{1}+b_{2}\right)$ is the slope that represents how the agreement between the two predictor variables (eg, exercisers perception and fitness trainers' use of autonomy-supportive behaviors) relate to the outcome (eg, autonomy satisfaction). The response surface $a_{2}\left(b_{3}+b_{4}+b_{5}\right)$ is the curvature and shows whether this relationship (between the agreement of the behaviors and the outcome) is linear or non-linear, that is, if the outcome differs depending on whether the ratings are high and in agreement or low and in agreement. The second line runs diagonally from the left to the right corner of the graph. It is called the "line of incongruence" where the slope is reflected by the response surface $a_{3}\left(b_{1}-b_{2}\right)$ and $a_{4}$ $\left(b_{3}-b_{4}+b_{5}\right)$. Similar to the line of perfect agreement, the curvature shows how discrepancies between predictors (eg, exercisers perceiving higher levels on one behavior compared with fitness trainers' perception of the same behavior) relate to the outcome and if the slope direction matters.

\section{3 | RESULTS}

\section{1 | Preliminary analysis}

The descriptive statistics and composite reliability coefficients of all study variables are summarized in Table 1. Examining mean values, fitness trainers' use of need-supportive, and need-thwarting behaviors were higher compared with the means of exercisers' perception of the same behaviors. Means for exercisers' psychological need satisfaction were above midpoint and higher compared with need frustration. No univariate outliers were found. Missing data were found in seven exercisers (less than 3\% of data), and thus, data were imputed using regression procedures.

See Table 2 for the correlations among study variables. Fitness trainers' use of need-supportive behaviors was rarely correlated with exercisers' perception of need-supportive behaviors, except for relatedness support. Additionally, only fitness trainers' use of need-thwarting behaviors was positively correlated with exercisers' psychological need frustration. The highest correlation coefficients were between exercisers' perceptions of need-supportive and need-thwarting behaviors, and their respective need (eg, perceived relatedness support and relatedness satisfaction, $r=.80, P<.001$ ).

\section{2 $\quad$ Frequencies of predictor discrepancies and agreement}

The average rates of predictor discrepancies and agreement are displayed in Table 3. The results support a fairly even distribution between the three groups, in which about one third of exercisers and fitness trainers are in agreement in competence thwarting, relatedness support, and relatedness thwarting behaviors. Overall, fitness trainers tend to over-report their behaviors, except in relatedness support. The discrepancy between fitness trainers' and exercisers' perceptions of interpersonal behaviors was larger than $10 \%$, thus indicating that RSA was warranted for analyzing the level of congruence in the data. 
TA B L E 1 Descriptive statistics and composite reliability scores of all study variables

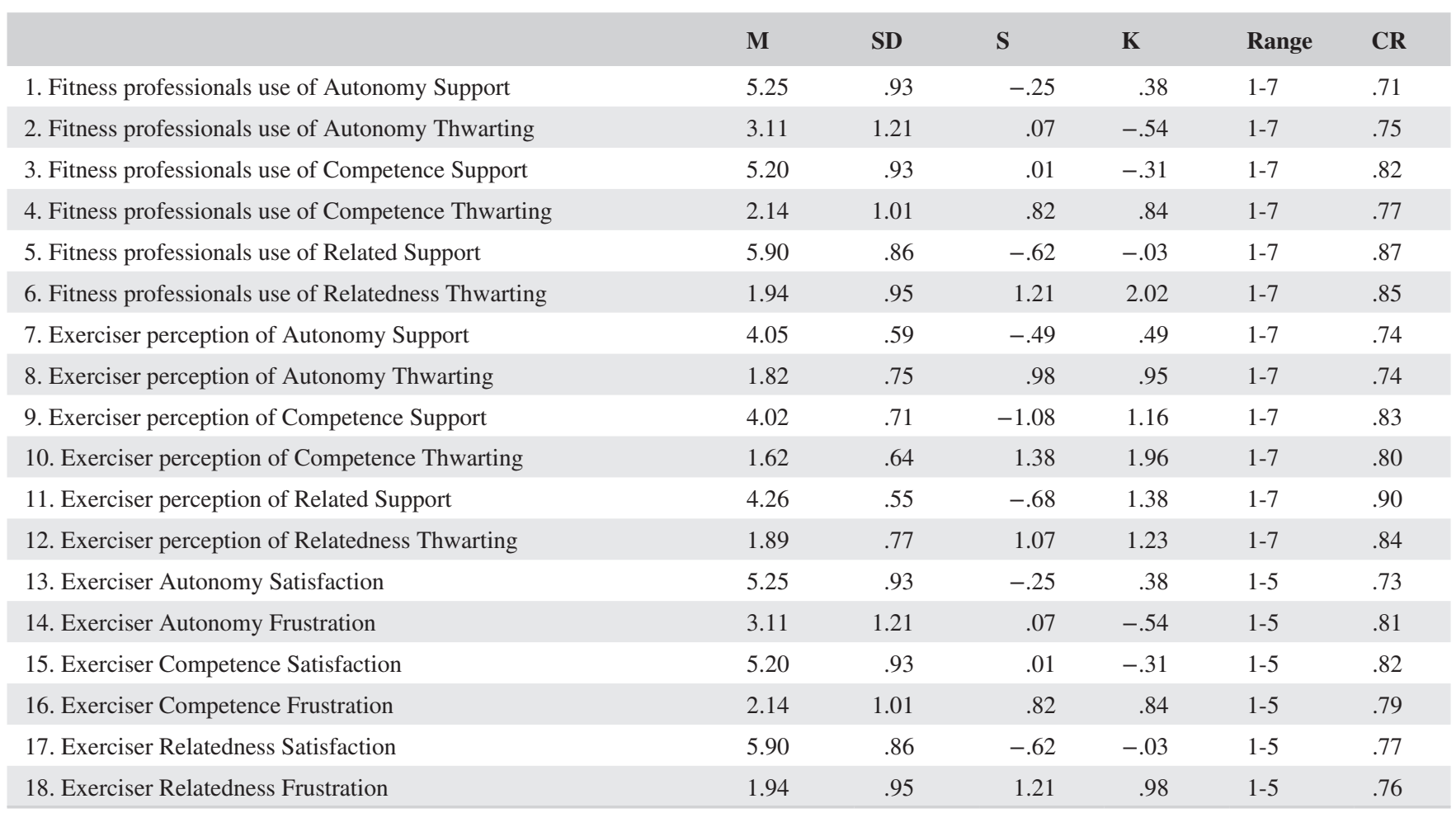

Abbreviations: $\mathrm{M}=$ mean; $\mathrm{SD}=$ standard deviation; $\mathrm{S}=$ skewness; $\mathrm{K}=$ kurtosis; $\mathrm{CR}=$ composite reliability.

\section{3 | Multilevel response surface analysis}

Results from the multilevel RSA analyses on exercisers' need satisfaction and need frustration are shown in Table 4. The results from all analyses supported that the regression models were significant, and the explained variance ranged from $11 \%$ (relatedness frustration) to $33 \%$ (competence satisfaction). Based on these findings, the surface test values were calculated for all six psychological needs.

Looking at the surface values, $a_{1}$ was significant in all models. This suggests that when fitness trainers' and exercisers' perceptions of interpersonal behaviors are in agreement, exercisers ratings on each need increases. As seen in Figure 1A (autonomy satisfaction) to If (relatedness frustration), the ratings of interpersonal behaviors increase along the line of perfect agreement, from the front corner to the back corner of the graph, where fitness trainers' and exercisers' perception of interpersonal behaviors are aligned (significant positive $a_{1}$ score). The lowest values are at the front of the graph, where the perceptions of interpersonal behaviors of both groups are low. As seen by the non-significance of the surface value test $a_{2}$, the curvature along the line of agreement showed that the influence of the perceived interpersonal behaviors on the respective need did not differ between high and low behaviors when exercisers and trainers were in agreement. That is, even when exercisers or trainers rated high on these behaviors compared to fitness trainers' and exercisers' perception, respectively, exercisers do not experience higher levels of need satisfaction or need frustration.
The response surface $a_{3}$ was negative and significant in five of the six models supporting that the direction of discrepancy between fitness professionals and exercisers was meaningful for exerciser in some need satisfaction and frustration. Specifically, a significant negative $a_{3}$ indicates that autonomy, competence, and relatedness satisfaction, and autonomy and relatedness frustration are higher when the discrepancy is such that exercisers' perception of interpersonal behaviors is higher than fitness trainers' rated behaviors than vice versa. The graphs depict these results, showing that at the left corner of the graph where exercisers' perceptions are high, combined with fitness trainers' low perceptions, need satisfaction and need frustration are still relatively high. Whereas at the right corner of the graph where fitness trainers' perception of interpersonal behaviors is low combined with exercisers' rating high on the same behaviors, the outcomes are still low. Finally, the results show a positive and significant response surface $a_{4}$ on autonomy and relatedness satisfaction and competence frustration; that is, this outcome increases more sharply as the degree of discrepancy increases (see Figure 1F of how the surface is convex). These results show that the effects of need satisfaction and need frustration are higher when the discrepancy between the respective behaviors ratings increases.

Last, the variance scores were relatively small or trivial, ranging from .000 to .182 . Thus, it seems that the interactions between trainers-exercisers do not vary significantly between gym facilities. As such, this suggests we could discuss each 


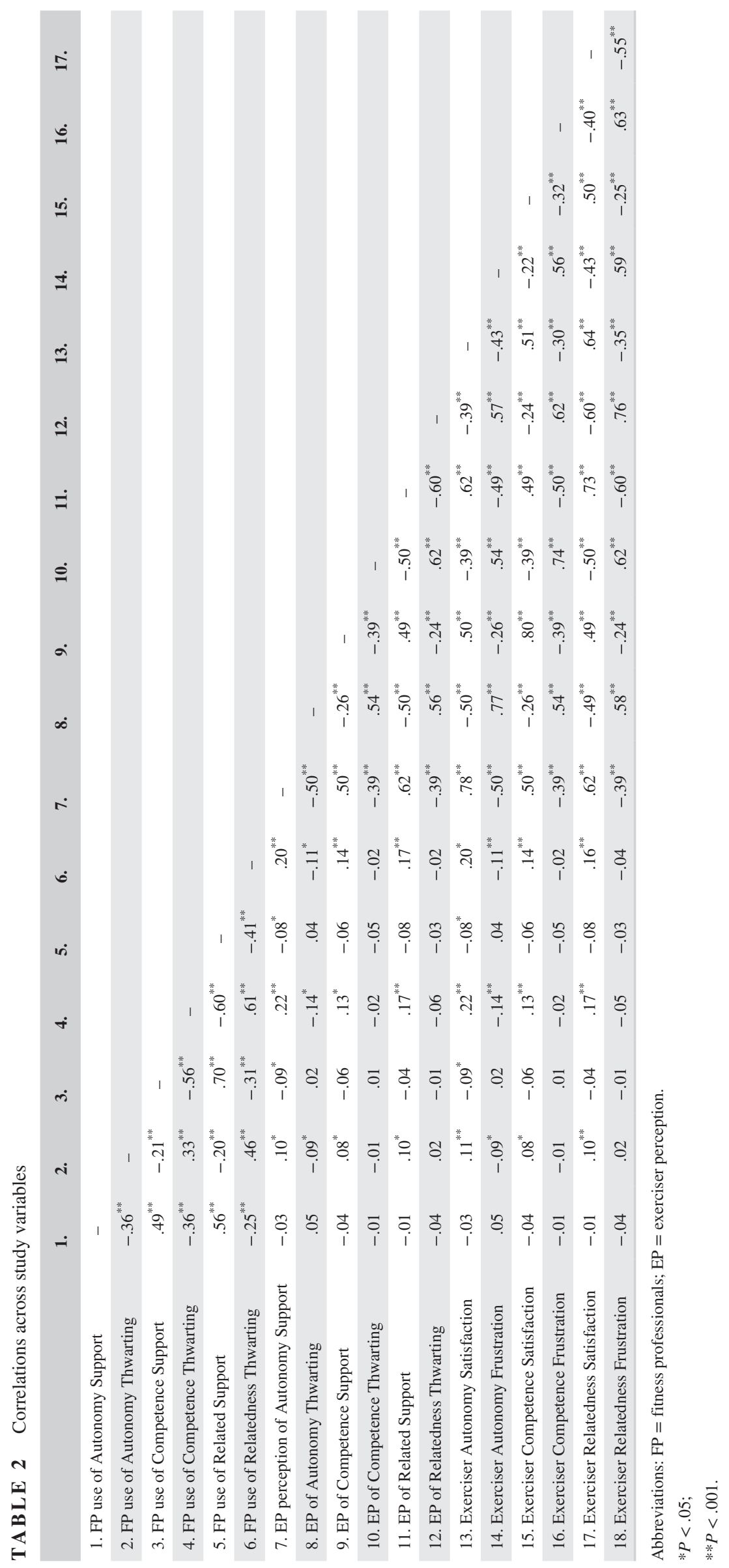


TA B L E 3 Frequencies of predictor discrepancies and agreement

\begin{tabular}{|c|c|c|c|}
\hline Groups & $\%$ & Mean fitness professionals & Mean exercisers \\
\hline Under-report (fitness professional > exerciser) & 37.0 & 4.54 & 6.05 \\
\hline Agreement (fitness professional $=$ exerciser) & 25.4 & 5.01 & 5.16 \\
\hline Over-report (fitness professional $<$ exerciser) & 37.6 & 5.69 & 4.61 \\
\hline \multicolumn{4}{|l|}{ Autonomy Thwarting } \\
\hline Agreement (fitness professional $=$ exerciser) & 27.4 & 3.84 & 3.62 \\
\hline Over-report (fitness professional $<$ exerciser) & 37.2 & 4.34 & 2.33 \\
\hline \multicolumn{4}{|l|}{ Competence Support } \\
\hline Under-report (fitness professional > exerciser) & 33.9 & 5.86 & 6.52 \\
\hline Under-report (fitness professional > exerciser) & 30.9 & 1.28 & 2.64 \\
\hline Agreement (fitness professional $=$ exerciser) & 33.5 & 1.49 & 1.43 \\
\hline Over-report (fitness professional $<$ exerciser) & 35.6 & 2.46 & 1.17 \\
\hline \multicolumn{4}{|l|}{ Relatedness Support } \\
\hline Under-report (fitness professional > exerciser) & 36.0 & 5.17 & 5.93 \\
\hline Agreement (fitness professional $=$ exerciser) & 30.5 & 5.74 & 5.32 \\
\hline Over-report (fitness professional $<$ exerciser) & 33.5 & 6.27 & 4.65 \\
\hline \multicolumn{4}{|l|}{ Relatedness Thwarting } \\
\hline
\end{tabular}

Note: Under-report $=$ fitness professionals displayed lower scores compared with exercisers perception; agreement $=$ fitness professionals and exerciser perception of interpersonal behaviors were similar; over-report $=$ fitness professionals displayed higher scores compared with exercisers' perception.

model in general, by taking in consideration the nested structure of the trainer-exerciser relationships within the gym facilities structure data.

\section{4 | DISCUSSION}

The purpose of present research was to examine the degree of agreement and discrepancy between self-reported and other-reported supportive and thwarting behaviors from fitness trainers and exercisers. The second aim was to examine the congruency effect between fitness trainers' and exercisers' perceptions of interpersonal behaviors on the exercisers' reported psychological need satisfaction and frustration. The current findings highlight that it is important to not only measure exercisers' perceptions of interpersonal behaviors, but also fitness trainers' reports of their own behaviors. In cases where fitness trainers and exercisers disagreed on the level of perceived needsupportive behaviors, results supported that fitness trainers rated themselves higher than exercisers perceived. This has a negative impact on exercisers' psychological needs, as fitness trainers could be ignoring their own behaviors and creating negative experiences for exercises, leading to negative outcomes.

\section{1 | Discrepancies and agreement in interpersonal behaviors}

To achieve objective 1, we explored the level discrepancy and agreement between exercisers' perception of fitness trainers' use of need-supportive and need-thwarting behaviors, and fitness trainers' own reports. To the best of our knowledge, only one study ${ }^{17}$ has assessed interpersonal behaviors between coaches and athletes. Overall, our results replicate the findings from this study in the exercise context. We found evidence to support that among the fitness trainers and exercisers, there were some fitness trainers who were in agreement with their exercisers, and others who either 


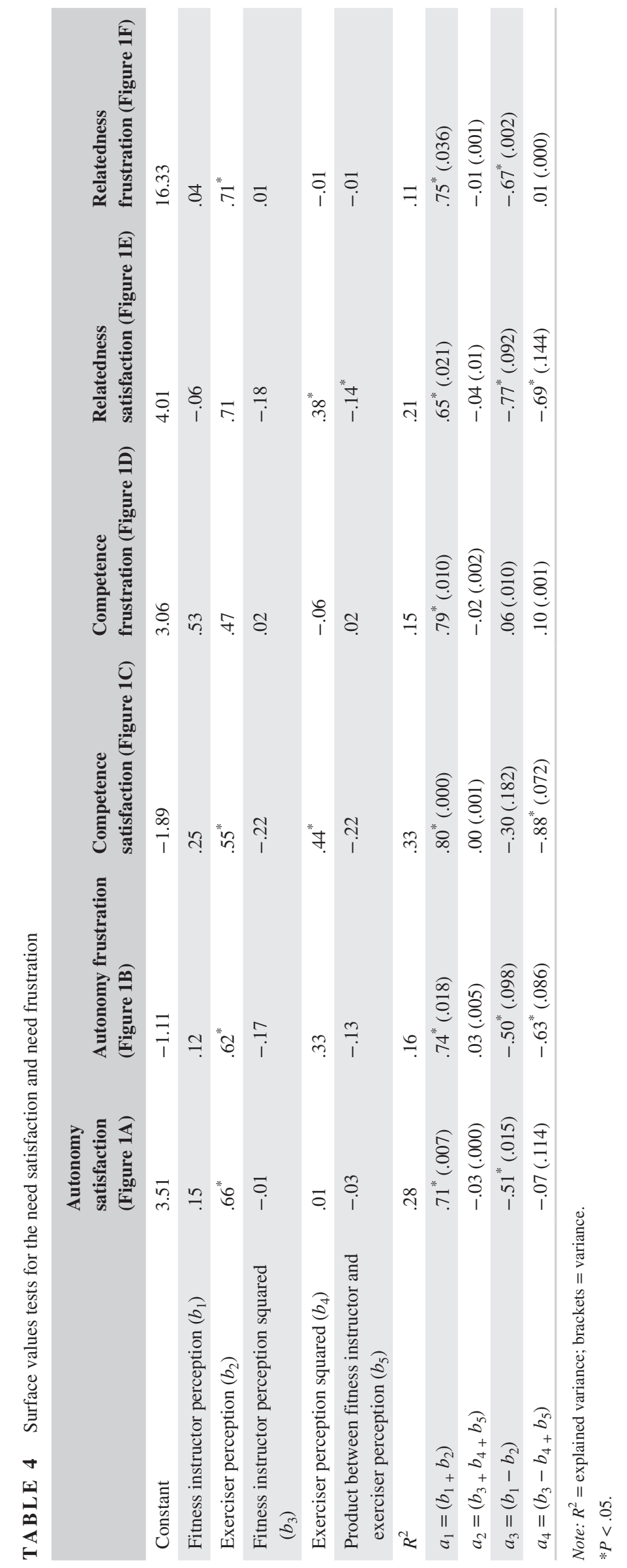


FIGURE 1 A, Perceived autonomy support and autonomy satisfaction. B, Perceived autonomy thwarting and autonomy frustration. C, Perceived competence support and competence satisfaction. D, Perceived competence thwarting and competence frustration. E, Perceived relatedness support and relatedness satisfaction. F, Perceived relatedness thwarting and relatedness frustration
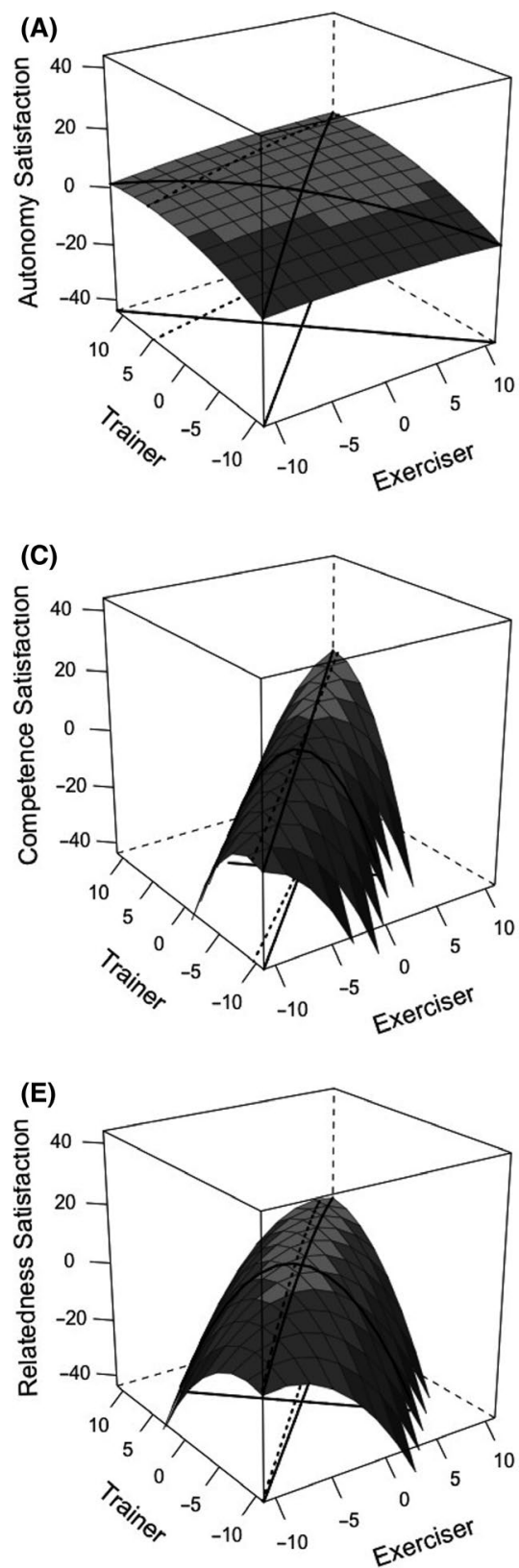
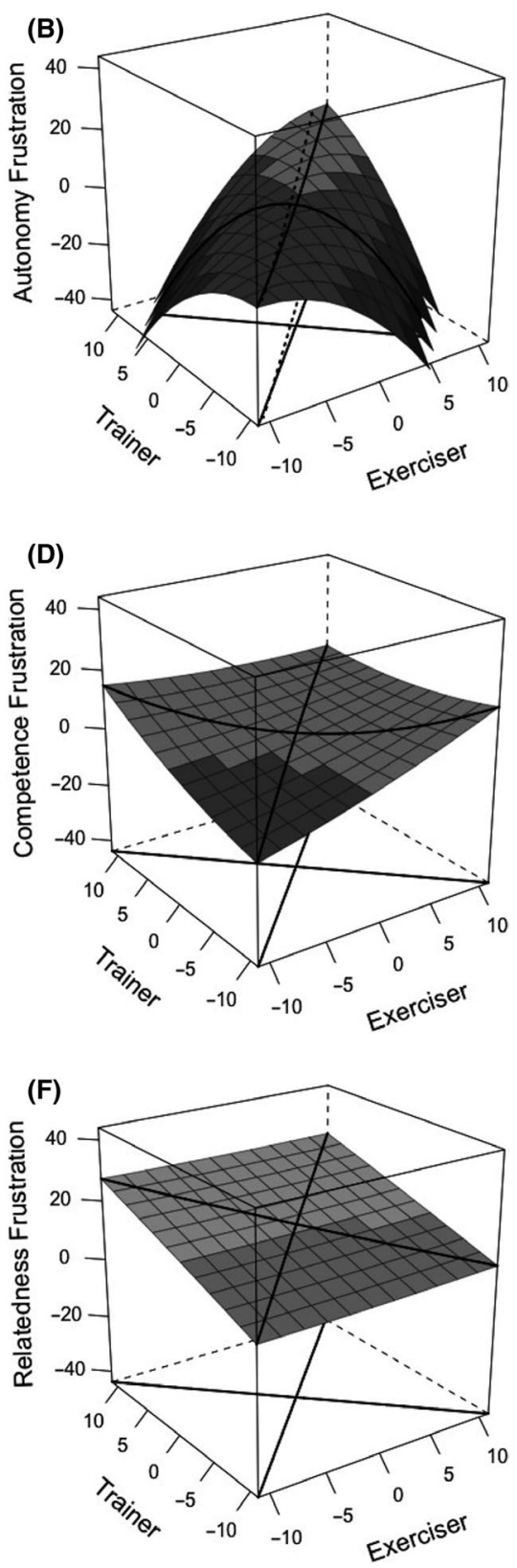

under-reported or over-reported their interpersonal behavior. Although we could not examine more specifically whether the results could be replicated for different pairs of men or women trainers and men or women exercisers due to sampling limitations, our results are consistent with most previous studies using a RSA approach. ${ }^{17,21}$ In sum, our results further highlight the importance of moving beyond one-way perspectives and considering both the perspectives of the fitness trainers and exercisers when examining need-supportive or need-thwarting behaviors in the context of physical exercise, since these behaviors are not always in agreement.

In terms of the frequencies, our observed percentages across behaviors are relatively balanced and similar to the proposed "benchmark" of $\sim 30 \%$ reported by previous research. ${ }^{17}$ The levels of agreement behaviors ranged from 25.4\% (autonomy support) to 33.5\% (competence support).
Under-reported behavior rates ranged from 30.9\% (competence thwarting) to $37 \%$ (autonomy support), which was very similar to over-reported behaviors. An interesting observation that may be specific to the present study is that we observed a high level of agreement between fitness trainers and exercisers on relatedness supportive behaviors. Although it is not clear why this occurred, future research could examine if this is specific to the participants' culture, the exercise context, or the interpersonal climate of specific gyms. Consistent with other recent studies that have reported that individuals in a position of authority (eg, coaches, teachers) have a tendency to over-report their positive behaviors ${ }^{17}$ or hypothesized that they might, ${ }^{23}$ we found that approximately $~ 30 \%$ of fitness trainers did over-report their behaviors. These results further highlight the need to control for this effect when conducting self-reported studies in interpersonal behaviors since both the 
over-reported and under-reported can negatively impact the validity and reliability of the findings. ${ }^{41}$

An interesting and intriguing question is what may lead individuals like fitness trainers to either over-report, under-report, or accurately report their interpersonal behaviors? Existing research has already found that psychological need satisfaction and frustration for individuals in positions of leadership are related to their reported interpersonal behavior $^{12,15,17,42}$ where psychological need frustration is associated with need-thwarting behavior. ${ }^{16}$ It may be possible that fitness trainers experience psychological need frustration (eg, when self-taught gym exercisers wish to train alone without professional support, or they experience pressure from the gym managers to get results with their clients) or have low psychological need satisfaction (eg, difficulty interacting with exercisers, or low recognition from exercisers and peers) and this may not only impact reported interpersonal behaviors, but also impact motivation to report interactions inaccurately. In other words, the multiplicity of situations that are inherent in gyms and wellness centers may not only influence how fitness trainers will interact with exercisers, but also how they will report these interactions. Given that social factors do not only impact the target of interpersonal behaviors (ie, exercisers) but likewise the transmitter (ie, fitness trainer), analyzing fitness trainers' determinants of their interpersonal behaviors could possibly shed some light on the reasons why some trainers may over-report or under-report their interpersonal behaviors. ${ }^{12,23}$

\subsection{Multilevel response surface analysis}

Regarding our second objective, the results support that the congruency effect between fitness trainers' reports of their own behavior and exercisers' perception of these behaviors is related to the exercisers' degree of need satisfaction and need frustration. Looking at the effects of autonomy, competence, and relatedness support on exercisers' respective need satisfaction, the present findings show that as the degree of agreement between fitness trainers and exercisers increases, so does the outcome. These results are similar to those found in sports ${ }^{17}$ and in the military ${ }^{43}$ as they provide further support for the importance of exploring the effects of self-other perceptions on an outcome. These results could be explained by the reciprocal effect between trainers and exercisers. As shown in the sport context, if a coach is supported by the context (eg, perceives their athletes to be motivated or perceives support from the administrators), they experience an increase in need satisfaction, which, in turn, leads to the use of need-supportive behaviors. ${ }^{17}$ Other studies found that, when trainers perceived that their exercisers were autonomously motivated, then trainers tend to feel autonomously motivated to engage with them, thus being more prone to engage in need-supportive behaviors, to provide rationale, to give positive feedback, and to engage in positive social interactions. ${ }^{15}$ Thus, it is therefore imperative that trainers are made aware of the potential positive impact of their engagement with the exercisers they supervise, so that they become aware of their own perceptions of interpersonal behaviors and motivation to prescribe exercise.

Higher ratings from exercisers in autonomy and relatedness support, compared with trainer's perceptions of the same behavior, were associated with higher scores on the outcome (see value for $a_{3}$ ). The current findings could be due to more emphasis on exerciser-exerciser interactions rather than trainer-exerciser interactions. As fitness trainers tend to communicate and help several exercisers, some of them may be prone to asking for help from other exercisers who could have some knowledge on exercise techniques. Another explanation for the current results may be the exercisers' willingness to train alone. In other contexts, such as sports or physical education, athletes and students are always guided by the coach or teacher. Contrarily, in gyms and health clubs, the variety of gym activities allows members to train what they want and how they want. Additionally, if the gym members do not want help from the fitness instructor, they can choose to train self-taught (eg, by seeing others, by exploring online videos).

Regarding the effects of need-thwarting interpersonal behaviors on exercisers' need frustration, in agreement with SDT, ${ }^{5}$ our results supported that when both fitness trainers and exercisers reported need-thwarting behaviors, exercisers reported increased psychological need frustration. Studies analyzing thwarting behaviors and need frustration in exercise context are scarce. Existing literature has shown that when exercisers perceive fitness trainers as endorsing need-thwarting behaviors, they experience high levels of need frustration. ${ }^{2}$ Thus, these exercisers are more likely to engage in exercise because of controlled reasons, resulting in less intentions to engage in exercise in the future. Interestingly, previous research assessing exercise instructors' use of need-thwarting behaviors found that when they felt that their needs were being frustrated, they reported higher rates of perceiving themselves as need-thwarting figures. ${ }^{11}$ Similar trends were found in the sport context, where coaches experiencing greater levels of autonomy, competence, and relatedness frustration were those who also reported higher endorsement of thwarting behaviors. ${ }^{16,17}$ In similar physical activity settings, studies ${ }^{6,44}$ have shown that perceived controlling behaviors were significantly associated with need frustration. Perceiving that the social environment actively thwarts our needs will ultimately lead to negative outcomes such as dissatisfaction or dropout. ${ }^{2,13}$ In agreement with Rocchi and Pelletier ${ }^{17}$ autonomy and relatedness frustration, and also competence and relatedness satisfaction were higher when the discrepancy between behavior ratings increased. These results reinforce the different impact of trainers and exercisers' perceptions of need-supportive and need-thwarting behaviors on exercisers' needs. As shown in the sport context, when a coaches are in 
a non-supportive or even controlling context (eg, that their athletes are not motivated or amotivated), they experience an increase in need frustration, which promotes controlled motivation for coaching and the use of need-thwarting behaviors. ${ }^{17}$ Current results reinforce previous literature ${ }^{45}$ that suggests that trainer-exerciser should be looked as a two-person (dyadic) relationship and that the quality of the interaction between (ie, trainer-exerciser and exerciser-trainer) is important to create effective interventions that promote long-lasting exercise adherence. Thus, current findings support that selfother perceptions matter and that the fitness trainer and the exerciser view-point should be accounted for when measuring interpersonal behavior and psychological needs.

\section{3 | Perspective}

Taken together, the present findings provide relevant practical implications for how two sources of interpersonal behaviors are related to basic psychological need experiences. From a research perspective, the current findings answer the calls for trainer-exerciser research to identify the congruency effect that can explain the prediction of need satisfaction and frustration. ${ }^{17}$ Based in SDT, ${ }^{5}$ our findings suggest that higher levels of need satisfaction and need frustration are expected to occur when exercisers' and fitness trainers' perceptions are in agreement on interpersonal behaviors. The level of agreement will allow for both the development of stronger relationships between trainers and exercisers, as well improved intentions to engage in exercise in the long-term. Previous literature has described the coach as being the dominant and controlling partner in the trainer-athlete dyad. ${ }^{45}$ Thus, it is possible that exercisers may derive greater social connections with fitness trainers when they display highly congruent interpersonal characteristics.

In applied practice, our findings may suggest that although many academic courses include exercise psychology and/or social sciences, such training courses may not be sufficient for those actually working in these domains. Fitness trainers who rate themselves highly may not be aware of their own behaviors and how they are perceived by those they train. This may lead them to rely mostly on their own assumptions of their behavior and may not take exercisers' feedback. As shown by Ntoumanis and colleagues, ${ }^{10}$ being perceived as need-supportive by exercisers is essential for exerciser need satisfaction. Thus, fitness trainers should be aware of their behaviors when engaging with exercisers, raising the question of whether the content of current leadership training teaches fitness trainers about the best practices for engaging with clients. Training interventions based in SDT could represent a promising avenue to improve the quality of exercisers experience.

Another way to increase behavior consciousness is to rely on videotaping fitness trainers' behaviors and letting them examine how they interact with exercisers. Looking at themselves when working with others might give trainers a new perspective, lead them to adopt more need-supportive behaviors, and possibly lead them to have a more balanced or accurate perception of their own interpersonal behaviors. If done in an informative climate, we think that fitness professionals could be evaluated regularly by exercisers, peers, and managers in terms of professional conduct. Triangulating these assessments could give important knowledge to fitness professionals about how they behave and how their behaviors are perceived by others. This may lead trainers to adapt their behaviors as a way to promote psychological need satisfaction.

\section{5 | CONCLUSION}

The findings in this study deepen our understanding of how the complex process of interpersonal communication in a trainer-exerciser dyad has an impact on exercisers' psychological need fulfillment. The results highlight the importance of positive and congruent perceptions of interpersonal behaviors as a clear indicator of high need satisfaction for exercisers. It is clear that fitness trainers' perceptions of interpersonal behaviors do not always align with how gym members perceive their behaviors. Thus, gym managers may want to develop supervisory training programs that enhance fitness trainers' self-awareness and also sensitize them to the perceptions of their exercisers.

Finally, multilevel RSA continues to demonstrate its power as a sophisticated statistical methodology to examine how two perspectives on interpersonal behaviors could be related, and how this relationship could have an impact on need satisfaction and need frustration. This approach is not only useful statistically to show that fitness professionals' use of need-supportive and need-thwarting behaviors do not always correspond to the behaviors perceived by exercisers, but it is also useful from a practical point of view to illustrate the implications of having behaviors that are perceived to be in agreement or in disagreement in a context like physical activity. This is crucial since exercisers, as well as fitness trainers, engage with and between each other, experiencing different levels of need fulfillment. Since psychological need satisfaction is related to positive outcomes, it is important that fitness trainers are aware of their own interpersonal behaviors in order to align their perceptions with those from exercisers.

\section{1 | Limitations}

A limitation of this study is that only need-based experiences were considered as outcomes of perceived interpersonal behaviors. Because those experiences are rather proximal outcomes of need-supportive and need-thwarting behaviors, future 
studies should assess more distal outcomes that follow from the satisfaction or the thwarting of basic psychological needs (eg, exercise persistence or adherence) and examine the congruency effect of two distinct but similar and commensurable constructs.

Given that exercisers deal with different fitness professionals during their weekly trainings, their perceptions of need-supportive and need-thwarting behaviors could have been seen as a more general view than a specific one. Thus, the agreement of behaviors might be moderated by the frequency of contact or overall level of experience with their main fitness trainer. Forthcoming studies should focus on one-on-one relationships, for example, personal trainers and their respective gym members. Another research avenue should examine differences in exercisers enrolled predominantly in group fitness classes with those enrolled in cardio-resistance training. Interesting results could emerge since different levels of exposure to the fitness trainers, as well as other fellow exercisers, could lead to distinct results.

Given the range representation in gender within this sample and the potential implications of exercising (eg, preferences, experience, intensity tolerance, levels of need fulfillment and need frustration), it would seem elucidative to consider covariates within RSA models in future studies. A dyadic RSA approach could be suitable to examine the congruency effect between members of the same gender or age groups.

Since this study is context-specific, future studies should examine similarities or differences in how agreement and discrepancies of interpersonal behaviors could predict how psychological needs are satisfied or frustrated in other contexts such as physical education. As illustrated in the present research, as well as in previous research, ${ }^{17}$ the levels of agreement between leaders and follower perceptions of interpersonal behaviors are related to increased psychological need satisfaction, but the reasons for the levels of agreement between trainers and exercisers are not well understood.

It is still not clear why some trainers may over-report or under-report the extent to which their interpersonal behaviors are need-supportive or need-thwarting. Future studies should analyze how the context (eg, administration support, exercisers perceived motivation) influences fitness trainers' need satisfaction and need frustration and their report of interpersonal behaviors that need-supportive and need-thwarting. Past research in the sport context has shown that lower levels support from colleagues or administrators and higher time constraints were predictors of psychological need frustration. ${ }^{12}$ Thus, it may be possible that the work context may also influence the extent to which fitness trainers over-report or under-report their interpersonal behaviors. Finally, it is possible that exercisers may be the ones that over-report or under-report the extent to which their trainers are need-supportive or need-thwarting in their interactions. Future studies should pay attention as well to exercisers' characteristics (eg, their tendency to be critical or positive of others, their motivational orientation, their cultural background, or their preference for a specific type of interpersonal behaviors) that could influence how they perceive their trainers or their expectations of them.

\section{CONFLICT OF INTEREST}

None to declare.

\section{ORCID}

Filipe Rodrigues iD https://orcid.org/0000-0003-1327-8872

Henrique P. Neiva iD https://orcid.org/0000-0001-9283-312X

Diogo S. Teixeira iD https://orcid.org/0000-0003-4587-5903

Luis Cid iD https://orcid.org/0000-0001-8156-3291

Diogo Monteiro (iD https://orcid.org/0000-0002-7179-6814

\section{REFERENCES}

1. Teixeira P, Carraca E, Markland D, Silva M, Ryan R. Exercise, physical activity, and self-determination theory: a systematic review. Int J Beha Nutr Phys Act. 2012;9:78.

2. Rodrigues F, Teixeira DS, Neiva HP, Cid L, Monteiro D. The bright and dark sides of motivation as predictors of enjoyment, intention, and exercise persistence. Scand J Med Sci Sports. 2020;30:787-800.

3. Sibley BA, Bergman SM. What keeps athletes in the gym? Goals, psychological needs, and motivation of CrossFit ${ }^{\mathrm{TM}}$ participants. Int J Sport Exer Psych. 2018;16:555-574.

4. Humberg S, Nestler S, Back M. Response surface analysis in personality and social psychology: checklist and clarifications for the case of congruence hypotheses. Soc Psych Person Sci. 2018;10:409-419.

5. Ryan R, Deci E. Self-Determination Theory. Basic Psychological Needs in Motivation, Development, and Wellness. New York, NY: Guildford Press; 2017.

6. Bartholomew K, Ntoumanis N, Ryan R, Bosch J, ThogersenNtoumani C. Self-determination theory and diminished functioning: the role of interpersonal control and psychological need thwarting. Persona Socl Psych Bull. 2011;37:1459-1473.

7. Vansteenkiste M, Ryan RM. On psychological growth and vulnerability: Basic psychological need satisfaction and need frustration as a unifying principle. J Psychother Integ. 2013;23:263-280.

8. Vansteenkiste M, Ryan RM, Soenens B. Basic psychological need theory: advancements, critical themes, and future directions. Mot Emo. 2020;44:1-31.

9. Warburton V, Wang J, Bartholomew K, Tuff R, Bishop K. Need satisfaction and need frustration as distinct and potentially co-occurring constructs: need profiles examined in physical education and sport. Mot Emo. 2020;44(1):54-66. https://doi.org/10.1007/ s11031-019-09798-2

10. Ntoumanis N, Thogersen-Ntoumani C, Quested E, Hancox J. The effects of training group exercise class instructors to adopt a motivationally adaptive communication style. Scand J Med Sci Sports. 2017;27:1026-1034

11. Rodrigues F, Pelletier L, Neiva H, Teixeira D, Cid L, Monteiro D. Initial validation of the Portuguese version of the Interpersonal Behavior Questionnaire (IBQ \& IBQ-Self) in the context of exercise: measurement invariance and latent mean differences. CurPsycho. 2019:1-12.

12. Rocchi M, Pelletier L, Cheung S, Baxter D, Beaudry S. Assessing need-supportive and need-thwarting interpersonal behaviours: the interpersonal behaviours questionnaire (IBQ). Person Indi Dif. 2017;104:423-433. 
13. Costa S, Ntoumanis N, Bartholomew K. Predicting the brighter and darker sides of interpersonal relationships: does psychological need thwarting matter? Motiv Emo. 2014;39:11-24.

14. Conroy DE, Coatsworth JD. Assessing autonomy-supportive coaching Strategies in Youth Sport. Psycho Sport Exer. 2007;8:671-684.

15. Rocchi M, Pelletier L. The antecedents of coaches' interpersonal behaviors: the role of the coaching context, coaches' psychological needs, and coaches' motivation. J Sport Exer Psycho. 2017;39:366-378.

16. Stebbings J, Taylor I, Spray C. Antecedents of perceived coach autonomy supportive and controlling behaviors: coach psychological need satisfaction and well-being. J Sport Exer Psycho. 2011;33:255-272.

17. Rocchi M, Pelletier L. How does coaches' reported interpersonal behavior align with athletes' perceptions? Consequences for female athletes' psychological needs in sport. Sport Exer Perf Psych. 2018;7:141-154.

18. Horn TS. Coaching Effectiveness in the Sport Domain. Advances in Sport Psychology. 3rd ed. Champaign, IL: Human Kinetics; 2008:239-267, 455-459.

19. Ntoumanis N, Guerrero MD, Gadeke C, Thøgersen-Ntoumani C. Do exerciser weight status and perceived motivation predict instructors' motivation and beliefs about the exerciser? A test of motivation contagion effects. Body Image. 2018;26:10-18.

20. Edwards J. Polynomial regression and response surface methodology. In: Ostroff C, Judge A, eds. Perspectives on Organizational Fit. San Francisco, CA: Jossey-Bass; 2007:361-372.

21. Gjesdal S, Stenling A, Solstad B, Ommundsen Y. A study of coach-team perceptual distance concerning the coach-created motivational climate in youth sport. Scandinavian J Med Sci Sports. 2018;29:132-143.

22. Bean C, Forneris T, Brunet J. Investigating discrepancies in program quality related to youth volleyball athletes' needs support. Psycho Sport Exer. 2016;26:154-163.

23. Ntoumanis N. A Self-Determination Theory Perspective on Motivation in Sport and Physical Education: Current Trends and Possible Future Research Directions. Champaign, IL: Human Kinetics; 2012:91-128.

24. Nestler S, Humberg S, Schönbrodt F. Response surface analysis with multilevel data: illustration for the case of congruence hypotheses. Psychol Methods. 2019;24(3):291-308. https://doi. org/10.1037/met0000199

25. Blanchard C, Amiot C, Perreault S, Vallerand R, Provencher P. Cohesiveness, coach's interpersonal style and psychological needs: their effects on self-determination and athletes' subjective well-being. Psychol Sport Exerci. 2009;10(5):545-551. https://doi. org/10.1016/j.psychsport.2009.02.005

26. Hollembeak J, Amorose A. Perceived coaching behaviors and college athletes' intrinsic motivation: a test of self-determination theory. J App Sport Psycho. 2005;17(1):20-36. https://doi. org/10.1080/10413200590907540

27. Rocchi M, Pelletier L, Desmarais P. The validity of the interpersonal behaviors questionnaire (IBQ) in sport. Measure Phys Educ Exer Sci. 2016;21:15-25.

28. Rodrigues F, Hair J, Neiva H, Teixeira D, Cid L, Monteiro D. The basic psychological need satisfaction and frustration scale in exercise (BPNSFS-E): validity, reliability, and fender invariance in Portuguese exercisers. Percept Motor Skills. 2019;126(5):949-972.

29. Chen B, Vansteenkiste M, Beyers W, et al. Basic psychological need satisfaction, need frustration, and need strength across four cultures. Mot Emo. 2015;39:216-236.
30. Gravetter F, Wallnau L. Essentials of statistics for the behavioral sciences. 8th ed. Wadsworth, Belmont, CA: Cengage Learning; 2014.

31. Raykov T, Gabler S, Dimitrov D. Maximal reliability and composite reliability: examining their difference for multicomponent measuring instruments using latent variable modeling. Struct Equ Mod. 2015;23:384-391.

32. Cham H, Reshetnyak E, Rosenfeld B, Breitbart W. Full information maximum likelihood estimation for latent variable interactions with incomplete indicators. Multi Beha Research. 2017;52:12-30.

33. Muthén L, Muthén B. Mplus User's Guide. Los Angeles, CA: Muthén \& Muthén; 2010.

34. Gibson C, Cooper CD, Conger JA. Do you see what we see? The complex effects of perceptual distance between leaders and teams. Develop Learn Org: An Int Journal. 2009;94(1):62-76.

35. Fleenor JW, McCauley CD, Brutus S. Self-other rating agreement and leader effectiveness. Lead Quart. 1996;7:487-506.

36. Atwater L, Ostroff C, Yammarino F, Fleenor J. Self-other agreement: does it really matter? Person Psycho. 2006;51(3):577-598.

37. Fleenor JW, Smither JW, Atwater LE, Braddy PW, Sturm RE. Self-other rating agreement in leadership: a review. Lead Quart. 2010;21:1005-1034.

38. Fox J. Applied Regression Analysis and Generalized Linear Models. CA: Sage; 2016.

39. Lacobucci D, Schneider MJ, Popovich DL, Bakamitsos GA. Mean centering helps alleviate "micro" but not "macro" multicollinearity. Beha Research Meth. 2016;48:1308-1317.

40. Preacher K, Zyphur MJ, Zhang Z. A general multilevel SEM framework for assessing multilevel mediation. Psychol Methods. 2010;15(3):209-233. https://doi.org/10.1037/a0020141

41. Bound J, Brown C, Mathiowetz N. Measurement error in survey data. In: Heckman JJ, Leamer EE, eds. Handbook of Econometrics. Elsevier; 2001:3705-3843.

42. Haerens L, Vansteenkiste M, Aelterman N, Van den Berghe L. Toward a systematic study of the dark side of student motivation: antecedents and consequences of teachers' controlling behaviors. In: Liu WC, Wang JCK, Ryan RM, eds. Building Autonomous Learners: Perspectives from Research and Practice using Self-Determination Theory. Singapore: Springer Singapore; 2016:59-81.

43. Atwater L, Yammarino F. Does self-other agreement on leadership perceptions moderate the validity of leadership and performance predictions? Person Psycho. 1992;45:141-164.

44. Jowett S, Adie J, Bartholomew K, Yang S, Gustafsson H, LopezJiménez A. Motivational processes in the coach-athlete relationship: a multi-cultural self-determination approach. Psycho Sport Exer. 2017;32:143-152.

45. Jackson B, Dimmock JA, Gucciardi DF, Grove JR. Personality traits and relationship perceptions in coach-athlete dyads: do opposites really attract? Psycho Sport Exer. 2011;12:222-230.

How to cite this article: Rodrigues F, Pelletier L, Rocchi M, et al. Trainer-exerciser relationship: The congruency effect on exerciser psychological needs using response surface analysis. Scand. J. Med. Sci. Sports. 2021;31:226-241. https://doi.org/10.1111/ sms.13825 